\title{
Primer registro e histología básica del anfípodo terrestre Talitroides topitotum (Amphipoda: Talitridae), introducido en las zonas montañosas de Heredia, Costa Rica
}

\author{
Jorge Alfaro-Montoya ${ }^{1}$ y Rodolfo Umaña Castro ${ }^{2}$ \\ 1. Estación de Biología Marina: Lic. Juan Bertoglia Richards, Escuela de Ciencias Biológicas, UNA, Puntarenas, Costa Rica; jorge.alfaro.montoya@una.cr \\ 2. Laboratorio de Análisis Genómico, Escuela de Ciencias Biológicas, UNA, Heredia, Costa Rica; rodolfo.umana.castro@una.cr
}

Recibido 11-III-2013 Corregido 29-IV-2013 Aceptado 14-V-2013

\begin{abstract}
First report and basic histology of the terrestrial amphipod, Talitroides topitotum (Amphipoda: Talitridae), introduced to the mountain region of Heredia, Costa Rica. This study documents for the first time the introduction of the terrestrial amphipod, Talitroides topitotum (Talitridae) to mountain regions of San Rafael and Barva, Heredia, Costa Rica, with predominance of juveniles and adult females with and without eggs. The species comes from Asia and could have been introduced associated with exotic plants. Basic aspects of histology are described, including the cellular structure of the main organs: heart, intestine, hepatopancreas, ovary, nerve cord, gill and oostegite. Adult females present massive formations of spongy connective tissue surrounding the digestive system and ovaries; oocytes are observed in different stages of vitellogenesis, producing few large oocytes.
\end{abstract}

KEY WORDS

Crustacea, Amphipoda, Talitridae, Talitroides topitotum, histology

\section{RESUMEN}

Este estudio documenta por primera vez la introducción del anfípodo terrestre, Talitroides topitotum (Talitridae) en diversas zonas montañosas de los cantones de San Rafael y Barva, Heredia, Costa Rica, con predominio de juveniles y hembras adultas con y sin huevos. La especie es de origen asiático y podría haber sido introducida asociada con plantas exóticas. Se describen aspectos básicos de la histología de la especie, incluyendo la estructura celular de los principales órganos: corazón, intestino, hepatopáncreas, ovario, cordón nervioso, branquia y oosteguito. Las hembras adultas presentan masivas formaciones de tejido conectivo esponjoso rodeando el sistema digestivo y ovarios; los oocitos aparecen en diversas fases de vitelogénesis, produciendo pocos oocitos de enorme tamaño.

PALABRAS CLAVE

Crustacea, Amphipoda, Talitridae, Talitroides topitotum, histología
Los reportes de anfípodos terrestres de América son escasos y recientes. Lindeman $(1989 ; 1990 ; 1991)$ desarrolló una investigación para clarificar la presencia de estos crustáceos en México y Centro América, incluyendo Costa Rica. Su estudio revela la presencia de la siguiente especie nativa en Costa Rica-Panamá: Cerrorchestia hyloraina (Lindeman, 1990), distribuida desde el Volcán Rincón de la Vieja (Guanacaste), Monte Verde (Puntarenas), Parque Nacional Braulio Carrillo (Heredia), Turrialba (Cartago), hasta El Llano, Panamá.

El anfípodo terrestre Talitroides topitotum (Burt, 1934) (Familia Talitridae) fue originalmente descrito en Sri Lanka y es reconocido como una especie cosmopolita introducida en regiones tropicales y templadas del planeta (Lindeman, 1990; Richardson, 1992). En América, se ha reportado su introducción en Estados Unidos de Norteamérica: Carolina del Sur y Florida (Biernbaum, 1980), en México: Oaxaca y Ciudad de México (Lindeman, 1990; Álvarez, Winfield \& Cházaro, 2000), y en Brasil: Guaratuba, Paraná (Lopes \& Masurani, 2004a), en el Estado de Espírito Santo (Eutrópio \& Krohling, 2013) y São Paulo (Matavelli, Uehara-Prado, Leite \& Freitas, 2009). Esta especie se establece en el estrato superficial de bosques con suelos ricos con materia en descomposición, de la que se alimentan (Lindeman, 1991). En Brasil, la especie fue introducida con la importación de árboles jóvenes y aparece asociada con 
áreas reforestadas con Eucalyptus (L'Heritier, 1789) (Ulian \& Mendes, 1988, Lopes \& Masunari, 2004a).

A pesar del carácter cosmopolita de la especie, su biología básica ha sido poco estudiada (Álvarez et al., 2000). En la Ciudad de México (2 475m), T. topitotum se ha establecido y su población se incrementa en número de individuos durante los meses de Junio a Octubre, reproduciéndose todo el año (Álvarez et al., 2000). En Brasil, se reportó un análisis morfométrico, distribución y biología reproductiva de una población de T. topitotum establecida en la región de Guaratuba, Paraná (270 m; Lopes \& Masunari, 2004a; 2004b; 2004c), así como aspectos de ecología de poblaciones en el estado de São Paulo (Matavelli et al., 2009).

La histología general de la especie, a nuestro conocimiento, no ha sido reportada, aunque otros estudios han evaluado aspectos histológicos de partes bucales, sistema digestivo y hepatopáncreas de anfípodos, incluyendo la familia Talitridae (Correia, Pereira, Costa \& Carrapico, 2002; Davolos, Pavesi, Accordi \& De Matthaeis, 2010). Los estudios histológicos proporcionan información importante sobre las adaptaciones específicas de las especies a nuevos ambientes (Davolos et al., 2010) y permite analizar cambios morfológicos producto de exposición a tóxicos ambientales (Correia et al., 2002). En particular, la familia Talitridae ofrece una perspectiva única para el estudio de cambios evolutivos asociados con la colonización terrestre (Johnston, Johnston \& Richardson, 2004). La presente investigación es el primer reporte de introducción de $T$. topitotum en Costa Rica, su presencia en la región geográfica de estudio se complementa con observaciones histológicas básicas de la especie.

\section{METODOLOGÍA}

\section{Captura y procesamiento de muestras}

Se realizaron búsquedas puntuales de anfípodos terrestres durante el 2012-2013, mediante trampas de agua $(14 \mathrm{~cm}$ diámetro) nocturnas y remoción-filtración de sustratos húmedos de bosques, en diversas regiones de las montañas del cantón de San Rafael de Heredia $\left(10^{\circ} 00^{\prime} \mathrm{N}, 84^{\circ} 06^{\prime} \mathrm{O}\right)$ : Monte de la Cruz (1 862m), Las Chorreras (1650m), Los Ángeles Centro (1496m), Concepción (1490m), el cantón de Barva $\left(10^{\circ} 01^{\prime} \mathrm{N}, 84^{\circ} 07^{\prime} \mathrm{O}\right)$ : Santa Lucía (1 230m) y San José de la Montaña (1937m), y Heredia Centro $\left(9^{\circ} 59^{\prime} \mathrm{N}, 84^{\circ} 07^{\prime} \mathrm{O} ; 1155 \mathrm{~m}\right)$. Los ejemplares colectados fueron preservados en etanol 70\% y la identificación se basó en las claves dicotómicas de Lindeman (1989) y el reporte de Hou \& Li (2003). La longitud total (L.T.) de los individuos se midió desde el extremo anterior del rostro hasta el extremo posterior del telson, mediante digitalización a escala utilizando escaneado convencional; adicionalmente se registró la abundancia relativa de juveniles ( $<7 \mathrm{~mm}$ L.T.), adultos y proporción de hembras con y sin huevos.

Complementariamente, animales previamente identificados de T. topitotum fueron colectados en trampas de aguas nocturnas, preservados en solución Davidson por $24 \mathrm{~h}$ y almacenados en etanol $50 \%$ para su procesamiento histológico, según Bell \& Lightner (1988). Los anfípodos fueron montados en parafina, seccionados longitudinalmente y teñidos con hematoxilina-eosina por la Empresa Andrómeda, San José, Costa Rica. La especie nativa, $C$. hyloraina fue colectada en Río Macho $\left(9^{\circ} 46^{\prime} \mathrm{N}, 83^{\circ} 50^{\prime} \mathrm{O}\right.$; $1580 \mathrm{~m})$, Orosi, Cartago, como referencia diagnóstica. El material muestreado se depositó en la colección del Museo de Zoología, Escuela de Biología, UCR con las accesiones MZUCR 3032-01 (Talitroides topitotum) y MZUCR 3033-01(Cerrorchestia hyloraina).

\section{RESULTADOS}

Los sitios muestreados con presencia de la especie corresponden a zonas húmedas con hojarasca o materia orgánica en descomposición. Las zonas positivas se caracterizaron por una abundancia alta de individuos, lo que facilitó las capturas. Las muestras consistieron principalmente de juveniles y hembras adultas con y sin huevos; la captura de machos fue casi nula. De todos los ejemplares colectados, solamente se capturó un macho adulto, en una región no incluida en este trabajo. La abundancia relativa de estos componentes poblacionales se registró para las muestras puntuales colectadas en las diversas localidades (Cuadro 1).

El diagnóstico de T. topitotum se basó en las siguientes características, según Lindeman (1989) y Hou \& Li (2003): adultos sin dimorfismo sexual, machos y hembras con gnatopodios 1-2 simples, la longitud de la antena 1 casi alcanza la longitud del pedúnculo de la antena 2, el tercer segmento del pedúnculo de la antena 1 es largo, los oosteguitos del gnatopodio 2 en hembras ovígeras con seis espinas marginales, la espina inter-ramal de los urópodos 1 muy larga, con punta levemente doblada, la rama externa de los urópodos 2 sin espinas dorsales en el margen, el pedúnculo de los urópodos 3 con tres espinas laterales fuertes, los terceros pleópodos son vestigiales (Fig. 1).

La especie nativa, C. hyloraina, colectada en Rio Macho, Cartago, presenta características diagnósticas muy diferentes. En resumen, es una especie con marcado dimorfismo sexual, la antena 1 no sobrepasa el segmento 4 del pedúnculo de la antena 2, el gnatopodio 1 presenta 
CUADRO 1

Presencia de anfípodos terrestres (Familia Talitridae) en Heredia y Cartago, Costa Rica

\begin{tabular}{|c|c|c|c|c|c|c|}
\hline \multirow{2}{*}{$\begin{array}{l}\text { Provincia } \\
\text { Heredia }\end{array}$} & \multirow{2}{*}{$\begin{array}{c}\text { Región geográfica } \\
\text { Monte de la Cruz }\end{array}$} & \multirow{2}{*}{$\begin{array}{c}\begin{array}{c}\text { Altitud } \\
\text { (msnm) }\end{array} \\
1862\end{array}$} & \multirow{2}{*}{$\begin{array}{c}\text { Especie } \\
\text { Talitroides topitotum }\end{array}$} & \multicolumn{3}{|c|}{$\begin{array}{c}\text { Abundancia relativa (\%)* } \\
\text { Juveniles Hembras } H \text {. ovígeras }\end{array}$} \\
\hline & & & & 75 & 100 & 0 \\
\hline & Las Chorreras & 1650 & Talitroides topitotum & 67 & 100 & 33 \\
\hline & Los Ángeles Centro & 1496 & Talitroides topitotum & 83 & 100 & 33 \\
\hline & Concepción & 1490 & Talitroides topitotum & 57 & 100 & 0 \\
\hline & San Rafael Centro & 1270 & Especie no confirmada & \multicolumn{3}{|c|}{ Referencia verbal } \\
\hline & Santa Lucía & 1230 & Talitroides topitotum & 57 & 100 & 0 \\
\hline & San José de la Montaña & 1937 & Muestreo negativo & & & \\
\hline & Heredia Centro & 1155 & Muestreo negativo & & & \\
\hline Cartago & Río Macho, Orosi & 1580 & Cerrorchestia hyloraina & & & \\
\hline
\end{tabular}

*Abundancia de juveniles menores de $7 \mathrm{~mm}$ longitud total y de adultos mayores de $7 \mathrm{~mm}$.

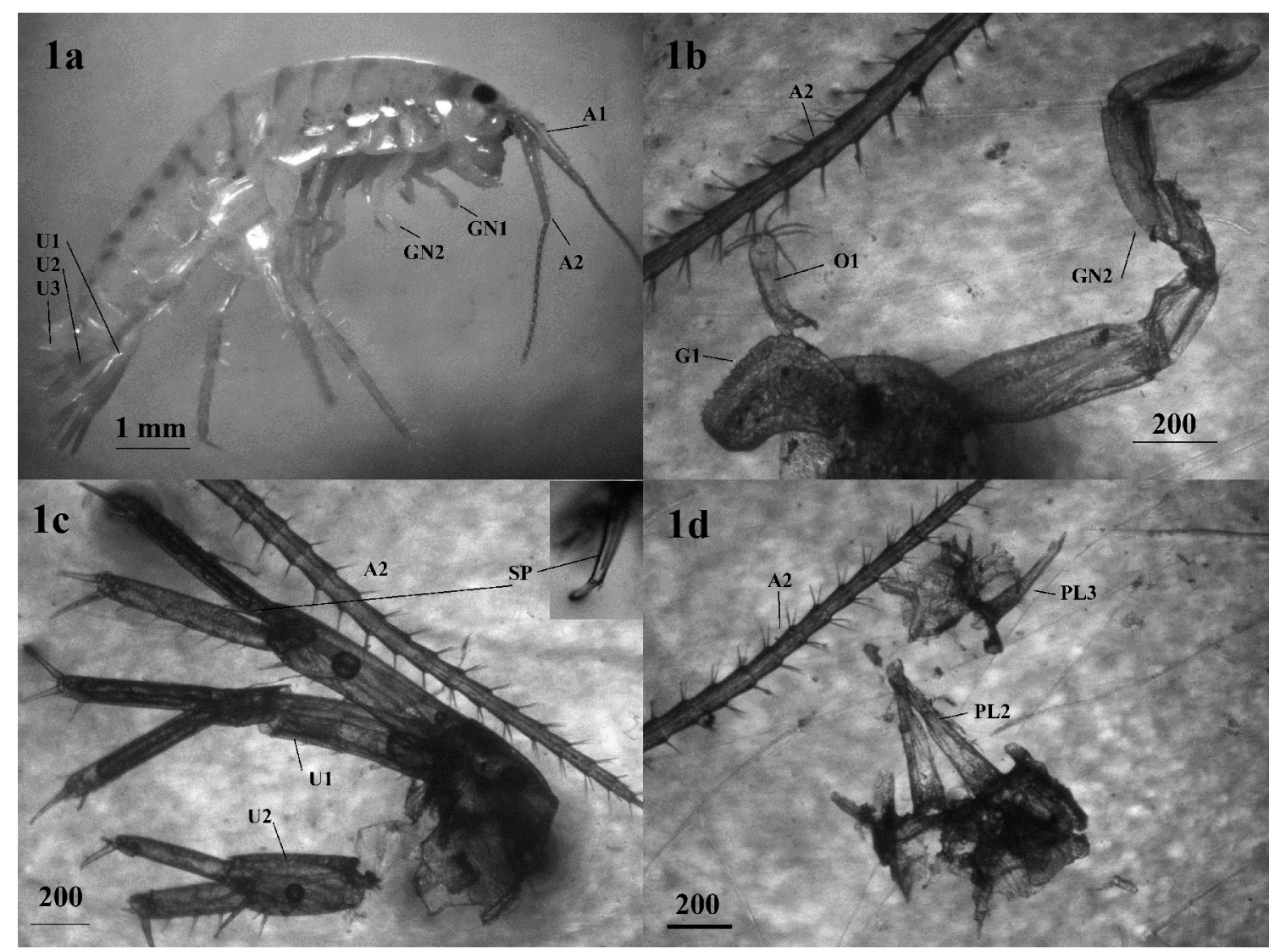

FIG. 1. Algunas características diagnósticas de Talitroides topitotum (Talitridae), introducida en las zonas montañosas de Heredia, Costa Rica. Foto 1a: hembra. A1: primeras antenas, A2: segundas antenas, G1: branquia 1, GN1: gnatopodio 1, GN2: gnatopodio 2, 01: oosteguito 1, U1-2-3: primeros, segundos y terceros urópodos, PL2-3: segundos y terceros pleópodos, SP: espina inter-ramal de urópodos 1. Escalas: $1 \mathrm{~b}-\mathrm{c}-\mathrm{d}=\mu \mathrm{m}$. 
vellosidades en mero, carpo y propodio, la rama interna de los urópodos 2 con una sola fila de espinas dorsales marginales, el pedúnculo de los urópodos 3 con 1-2 espinas laterales. Los terceros pleópodos son de menor tamaño que los anteriores. Los oosteguitos del gnatopodio 2 en hembras ovígeras con muchas espinas marginales (> 10; Fig. 2).

El tamaño máximo colectado de $T$. topitotum fue de $12 \mathrm{~mm}$ de longitud total. Las diversas zonas del cantón de San Rafael resultaron positivas con presencia de la especie, y en el cantón de Barva, la especie aparece en la zona de Santa Lucía, pero no fue posible obtener individuos, ni referencias verbales sobre su existencia, en el distrito de San José de la Montaña. En Heredia, cantón central, tampoco se obtuvieron ejemplares de la especie. La especie aparece desde los $1232 \mathrm{~m}$ hasta los $1862 \mathrm{~m}$ (Cuadro 1).

La histología básica de T. topitotum revela la disposición típica de órganos corporales de los anfípodos, indicando la estructura y localización de tejidos y órganos en corte longitudinal a nivel medio del torax (Fig. 3). Dorso-ventralmente se observa la cutícula, la epidermis, bandas de músculo dorso-lateral, el corazón, gónadas, tejido conectivo esponjoso, tubo digestivo y hepatopáncreas. La cutícula muestra claramente 2 estratos típicos: la epicutícula densa y delgada, la exocutícula ancha; la endocutícula no se observa. El corazón es tubular con un epitelio simple. El tubo digestivo anterior está recubierto por un estrato simple de epitelio cuboidal y en el lumen aparece materia alimenticia.

Los ovarios (Fig. 4) presentan pocos oocitos en fases diversas de vitelogénesis, cada oocito aparece rodeado de un estrato de células foliculares delgadas. Oocitos en vitelogénesis temprana son fuertemente basofílicos (tinción azul-púrpura) y en vitelogénesis avanzada aparecen con glóbulos citoplasmáticos acidofílicos (tinción rosa) y núcleos irregulares basofílicos; las dimensiones máximas medidas de oocitos fueron de $900 \mu \mathrm{m}$ largo x $250 \mu \mathrm{m}$

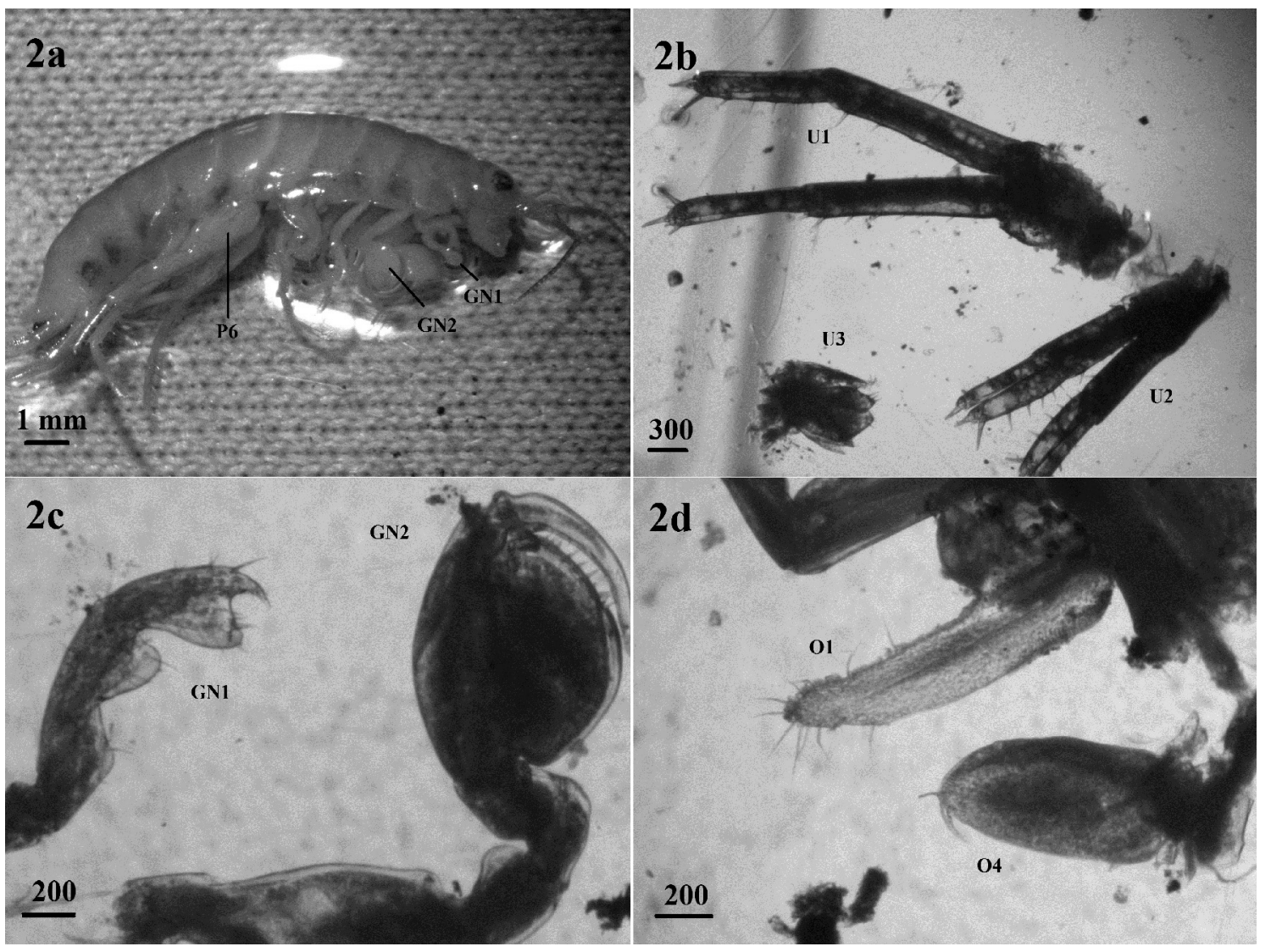

FIG. 2. Características diagnósticas de la especie nativa, Cerrorchestia hyloraina (Talitridae), colectada en Río Macho, Orosi, Cartago, Costa Rica. Foto 2a: macho. GN1: gnatopodio 1 del macho, GN2: gnatopodio 2 del macho, 01: oosteguito 1, 04: oosteguito 4, U1-2-3: primeros, segundos y terceros urópodos, P6: pereiópodo 6, PL2-3: segundos y terceros pleópodos. Escalas: $2 \mathrm{~b}-\mathrm{c}-\mathrm{d}=\mu \mathrm{m}$. 


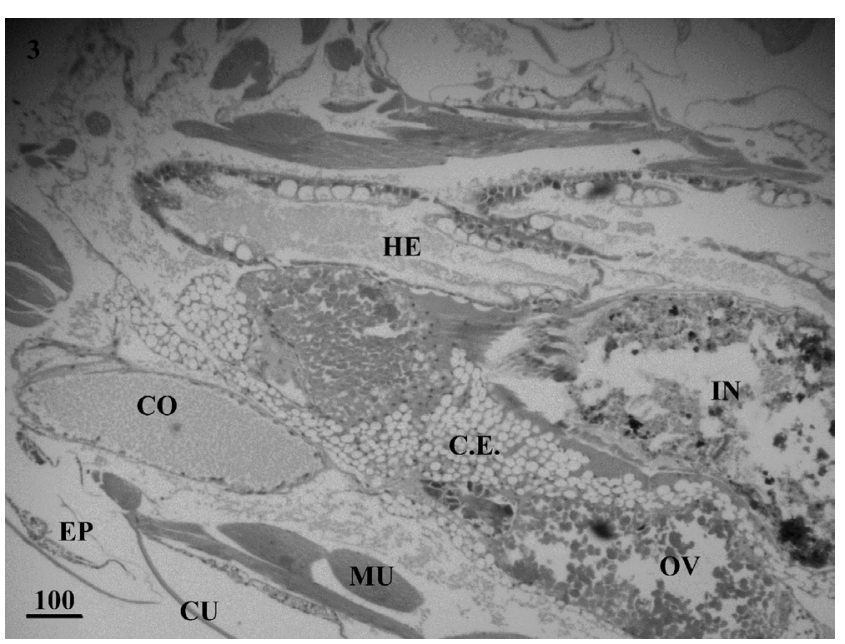

FIG. 3. Organización corporal de tejidos y órganos en la región media longitudinal de Talitroides topitotum. C.E.: conectivo esponjosos, CO: corazón, CU: cutícula, EP: epidermis, HE: hepatopáncreas, IN: intestino, MU: banda de músculo dorso-lateral, OV: ovario. Escala: $\mu \mathrm{m}$.

ancho. Las bandas musculares dorso-laterales inter-segmentos (Fig. 4) aparecen en pares a ambos lados del dorso, en corte transversal (no se muestra la figura).

El hepatopáncreas (Fig. 5) está formado por túbulos, cuyas zonas apicales contienen células indiferenciadas basofílicas (células embrionarias: C.E.); la región media presenta células diferenciadas, incluyendo células de absorción-almacenamiento (células R), caracterizadas por la presencia de pequeñas vacuolas citoplasmáticas, y células fibrosas (células F), con núcleos grandes y un nucléolo prominente (no se observan en la figura). La zona proximal, adicionalmente presenta células secretoras (células B), con una vacuola grande. El diámetro medio de los túbulos es de aproximadamente $140 \mu \mathrm{m}$.

El cordón nervioso ventral (Fig. 6) muestra su típica organización ganglionar, con los ganglios interconectados por un cordón nervioso. Se distinguen dos tipos de células nerviosas. Las branquias y oosteguitos (Fig. 7) presentan una fina cutícula; el epitelio branquial es un estrato columnar simple acidofílico y el interior forma una laguna sanguínea para el intercambio gaseoso.

\section{DISCUSIÓN}

Nuestro reporte de la presencia de T. topitotum en Costa Rica y particularmente en las montañas de los cantones de San Rafael y Barva de Heredia, es a nuestro conocimiento la primera documentación para Centro América.

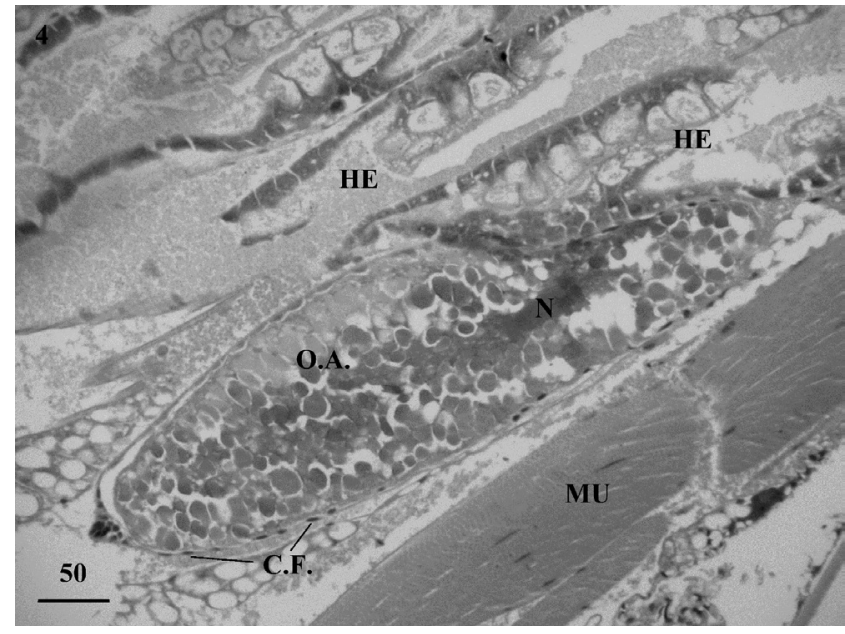

FIG. 4. Ovario con oocito en estado avanzado de vitelogénesis. C.F.: células foliculares, HE: hepatopáncreas, MU: músculo dorsolateral, N: núcleo, O.A.: oocito avanzado con glóbulos acidofílicos. Escala: $\mu \mathrm{m}$.

La introducción en Norte América y Sur América ha sido bien documentada (Biernbaum, 1980; Lindeman, 1990; Richardson, 1992; Álvarez et al., 2000; Lopes \& Masunari, 2004b; Eutrópio \& Krohling, 2013).

La presencia de la especie en diversas zonas montañosas de Heredia podría indicar que su introducción data de varios años en el pasado, tomando en cuenta su alta capacidad de colonización y la abundancia de especímenes observados en cada sitio positivo de muestreo; sin embargo, con la información disponible, no podemos definir ninguna hipótesis sobre el origen de su llegada al país. Las zonas habitadas por T. topitotum no corresponden con la presencia de C. hyloraina.

T. topitotum está bien establecida ya que hemos observado su presencia durante todo el año; en los meses de lluvias (junio-noviembre) sus actividades nocturnas aumentan, generando masivas mortalidades por desecación en áreas residenciales. La escasez de machos en las recolecciones parece indicar una mayor proporción de hembras en las poblaciones monitoreadas, este parámetro ha sido medido en otros estudios de la región. En México, las proporciones de hembras siempre fueron mayores, hasta un máximo de 4:1 (Álvarez et al., 2000); en Brasil, no se capturaron machos durante todo el año de estudio (Lopes \& Masunari, 2004c; Matavelli et al., 2009).

La histología básica de $T$. topitotum revela adaptaciones a la vida terrestre muy particulares, que podrían ayudar a comprender las capacidades invasivas de esta especie. Los oocitos crecen hasta dimensiones considerables $(900 \mu \mathrm{m}$ largo $\times 250 \mu \mathrm{m}$ ancho) y los embriones alcanzan tallas ovales 


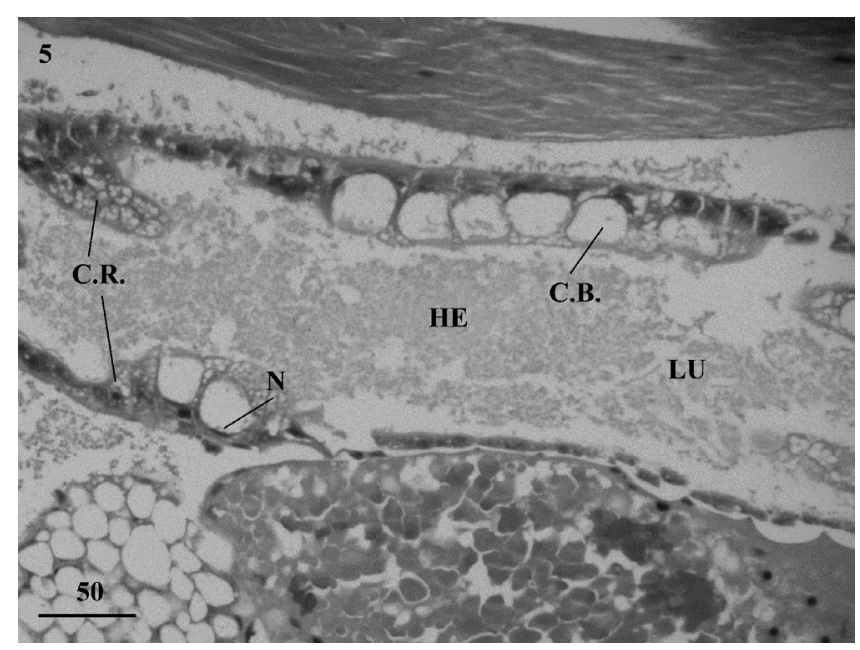

FIG. 5. Túbulo del hepatopáncreas en la región media y proximal. C.B.: células excretoras, C.R.: células de absorción, HE: hepatopáncreas, LU: lumen del túbulo, N: núcleo. Escala: $\mu \mathrm{m}$.

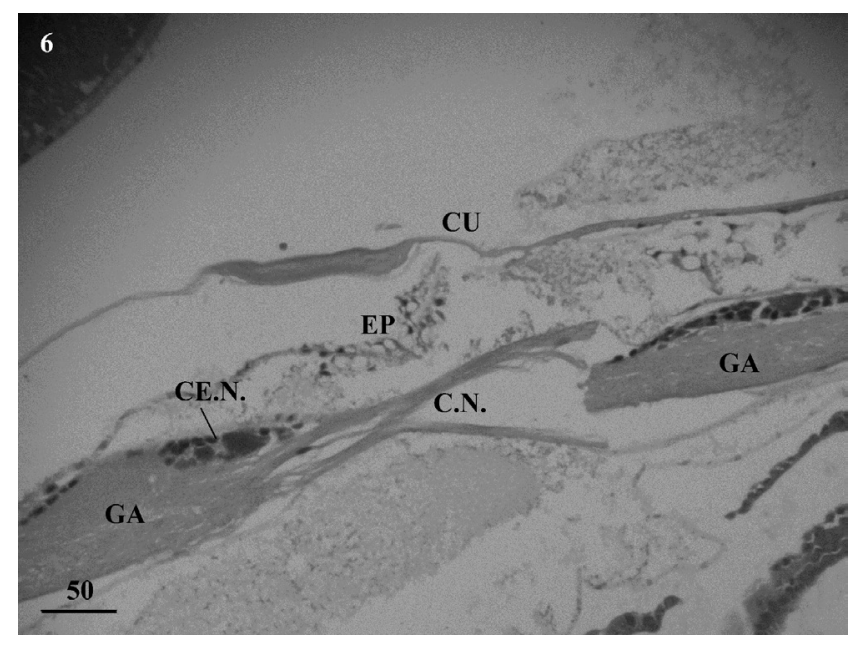

FIG. 6. Cordón nervioso ventral con detalle de la estructura ganglionar. C.N.: cordón nervioso, CE.N.: células nerviosas, CU: cutícula ventral, EP: epitelio, GA: ganglio. Escala: $\mu \mathrm{m}$.

que fluctúan entre 1 050-1 325 $\mu \mathrm{m} \times$ 875-1 025 $\mu \mathrm{m}$, muy superior a otros malacostráceos marinos, como el anfípodo Cystisoma (Guérin-Méneville, 1842) (Hyperiidea), cuyo tamaño máximo de oocitos es de 160 a $250 \mu \mathrm{m}$ de diámetro (Brusca, 1981); los camarones Litopenaeus (Decapoda: Dendrobranchiata: Penaeidae) liberan oocitos de $280 \mu \mathrm{m}$ de diámetro (Rojas \& Alfaro, 2007). Esta condición de producir oocitos grandes le permite a la especie incubar entre 1 y 9 huevos (Álvarez et al., 2000), a diferencia de Litopenaeus que logra liberar al mar, alrededor de 200,000 huevos (Vaca \& Alfaro, 2000), dependiendo del tamaño corporal.

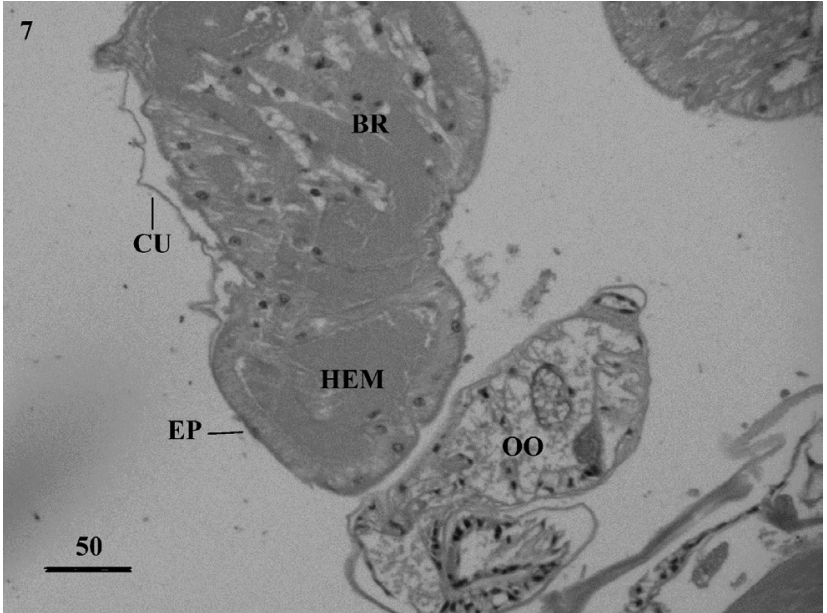

FIG. 7. Estructura de la branquia y oosteguito. BR: branquia, CU: cutícula, EP: epitelio, HEM: hemolinfa, OO: oosteguito. Escala: $\mu \mathrm{m}$.

La estructura del hepatopáncreas es similar a otros malacostráceos, caracterizada por la diferenciación progresiva de células desde la región distal hacia la región proximal. Los tipos celulares observados son los descritos previamente en Litopenaeus, Gammarus locusta (Linnaeus, 1758; Gammaridae) y Macarorchestia remyi (Schellenberg, 1950; Talitridae): células indiferenciadas (células E), células de almacenamiento (R), células fibrosas (F) y células secretoras (B) (Bell \& Lightner, 1988; Correia et al., 2002; Davolos et al., 2010). Sin embargo, el diámetro medio de los túbulos es mayor $(140 \mu \mathrm{m})$ al reportado para adultos de $G$. locusta $(50 \mu \mathrm{m})$, de tamaño similar (10-12mm; Correia et al., 2002).

Las bandas pares de músculo dorso-lateral son similares a las observadas en los anfípodos Cystisoma y M. remyi (Brusca, 1981; Davolos et al., 2010). Rodeando el tubo digestivo y el hepatopáncreas se forma tejido conectivo esponjoso, que está formado por adipocitos, que almacenan lípidos y proporcionan soporte mecánico. Las branquias son estructuras particularmente sensibles a la adaptación de vida terrestre; la histología revela un epitelio branquial columnar y una cutícula delgada en comparación con la cutícula corporal. Estas condiciones histológicas no le permiten a la especie sobrevivir en ambientes secos, por lo que son muy vulnerables a la desecación cuando realizan sus excursiones nocturnas fuera de los suelos húmedos.

Este primer reporte del anfípodo terrestre Talitroides topitotum contribuye con una mejor definición del rango de dispersión de la especie en América. Nuestras observaciones histológicas generales de la especie representan un primer aporte en este campo, que aunado a los estudios biológicos realizados en otras regiones geográficas, podrían ayudar a comprender sus capacidades adaptativas. 
Entre las iniciativas de nuestro grupo de investigación se plantea la ejecución de abordajes filogenéticos y filogeográficos basados en secuencias genéticas de ADN mitocondrial y nuclear para anfípodos terrestres en Costa Rica. Por otro lado, nuestra contribución con este reporte facilitaría a que otros científicos de la región complementen otras posibles introducciones de T. topitotum en el istmo centroamericano, acompañadas de investigaciones complementarias sobre biología y ecología de la especie.

\section{AGRADECIMIENTOS}

Los autores agradecen la colaboración del señor Eddy González Coto, Juan Ulloa Rojas y Iván Sandoval Hernández en la colecta de especímenes de C. hyloraina. Agradecemos en particular a los evaluadores anónimos de este manuscrito.

\section{REFERENCIAS}

Álvarez, F., Winfield, I., \& Cházaro, S. (2000). Population study of the landhopper Talitroides topitotum (Crustacea: Amphipoda: Talitridae) in central Mexico. Journal of Natural History, 34, 1619-1624.

Bell, T.A., \& Lightner, D.V. (1988). A Handbook of Normal Penaeid Shrimp Histology. Baton Rouge, Louisiana, USA:The World Aquaculture Society.

Biernbaum, C.K. (1980). Occurrence of the 'tramp' terrestrial amphipo Talitroides topitotum (Chevreux) and T. topitotum (Burt) (Amphipoda: Talitridae) in South Carolina. Brimleyana, 3, 107-111.

Brusca, G.J. (1981). On the anatomy of Cystisoma (Amphipoda: Hyperiidea). Journal of Crustacean Biology, 1(3), 358-375.

Correia, A.D., Pereira, A.L., Costa, M.H., Carrapico, F. (2002). Functional anatomy of the midgut gland of Gammarus locusta (Crustacea: Amphipoda). Journal of the Marine Biological Association of the UK, 82, 201-204.

Davolos, D., Pavesi, L., Accordi, F., \& De Matthaeis, E. (2010). Morphology and histology of the mouthparts and gut system of Macarorchestia remyi (Schellenberg, 1950)(Amphipoda, Talitridae). Zoologica Baetica, 21, 151-178.

Eutrópio, F.J., \& Krohling, W. (2013). First record of Amphipoda Talitroides topitotum (Burt, 1934) (Gammaridea, Talitridae) in the State of Espírito Santo, Brazil. Acta Scientiarum. Biological Sciences, 35(1), 37-39.

Hou, Z.E., \& Li, S. (2003). Terrestrial talitrid amphipods (Crustacea: Amphipoda) from China and Vietnam: studies on the collection of IZCAS. Journal of Natural History, 37, 2441-2460.
Johnston, M.D., Johnston, D.J., \& Richardson, A.M.M. (2004). Mouthpart and digestive tract structure in four talitrid amphipods from a translittoral series in Tasmania. Journal of the Marine Biological Association of the UK, 84, 717-726.

Lopes, O., \& Masunari, S. (2004a). Características morfométricas de Talitroides topitotum (Burt) (Crustacea, Amphipoda, Talitridae) na Serra do Mar, Guaratuba, Paraná, Brasil. Revista Brasileira de Zoologia, 21, 779-784.

Lopes, O., \& Masunari, S. (2004b). Distribuição de abundância de Talitroides topitotum (Burt) (Crustacea, Amphipoda, Talitridae) na área de entorno da Usina Hidroelétrica de Guaratuba, Serra do Mar, Guaratuba, Paraná, Brasil. Revista Brasileira de Zoologia, 21, 219-227.

Lopes, O., \& Masunari, S. (2004c). Biologia reprodutiva de Talitroides topitotum (Burt) (Crustacea, Amphipoda, Talitridae) na Serra do Mar, Guaratuba, Paraná, Brasil. Revista Brasileira de Zoologia, 21, 755-759.

Lindeman, D.H. (1989). Systematics and phylogeny of the landhoppers (Crustacea: Amphipoda: Talitridae) from Mexico and Central America. (Ph.D. Dissertation). Department of Biology, Carleton University, Ottawa, Ontario, Canada.

Lindeman, D.H. (1990). New terrestrial amphipods (Crustacea: Amphipoda:Talitridae) from Mexico and Central America. Canadian Journal of Zoology, 68, 2323- 2337.

Lindeman, D.H. (1991). Natural history of the terrestrial amphipod Cerrorchestia hyloraina Lindeman (Crustacea: Amphipoda: Talitridae) in a Costa Rican cloud forest. Journal of Natural History, 25, 623-638.

Matavelli, C., Uehara-Prado, M., Leite, F.P.P., \& Freitas, A.V.L. (2009). Some aspects of the population ecology of the exotic amphipod, Talitroides topitotum, in an Atlantic forest reserve in Brazil. Crustaceana, 82(2), 241-251.

Richardson, A.M.M. (1992). Altitudinal distribution of native and alien landhoppers (Amphipoda: Talitridae) in the Ko'olau Range, O'ahu, Hawaiian Island. Journal of Natural History, 26, 339- 352.

Rojas, E., \& Alfaro, J. (2007). In vitro manipulation of egg activation in the open thelycum shrimp Litopenaeus. Aquaculture, 264, 469-474.

Ulian, G.B., \& Mendes, E.G. (1988). Tolerances of a land amphipod, Talitrus (Talitroides) pacificus Hurley, 1955, towards temperature and humidity variations and immersion in water. Revista Brasileira de Biologia, 48(2), 179-187.

Vaca, A., \& Alfaro, J. (2000). Ovarian maturation and spawning in the white shrimp, Penaeus vannamei, by serotonin injection. Aquaculture, 182, 373-385. 
